
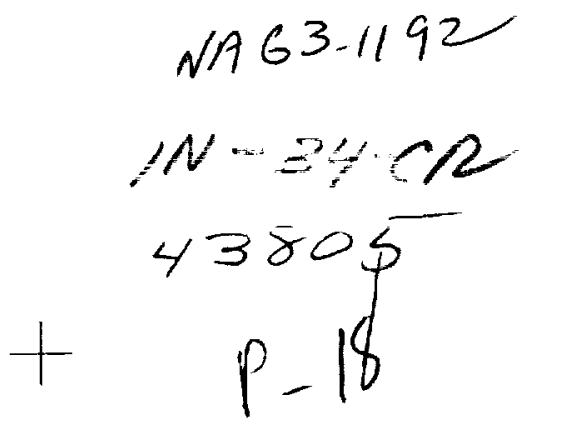

\title{
Calculation of Unsteady Linearized Euler Flows in Cascades Using Harmonically Deforming Grids
}

\author{
Kenneth C. Hall ${ }^{1}$ \\ William S. Clark2
}

\begin{abstract}
A method for calculating unsteady, inviscid, compressible flows in cascades is presented. Úsing the linearized Euler technique, the flow is decomposed into a steady or mean flow plus a harmonically varying small disturbance flow. The equations that describe the small disturbance flow are linear variable coefficient equations, and are solved using a pseudo-time time marching Lax-Wendroff technique. Unlike previous linearized methods, however, the solution is computed on a harmonically deforming computational grid that conforms to the motion of the vibrating airfoils. The mean flow and perturbation flow solutions are defined in the deforming coordinate system rather than in a coordinate system fixed in space. Hence, no extrapolation terms are required to implement the upwash boundary conditions at the airfoil surfaces significantly improving the accuracy of the method. For transonic flow calculations, unsteady shock motions are modelled using shock capturing. The unsteady loads due to the shock motion are then seen as pressure impulses. Representative computational results are presented for transonic channel flows and subsonic and transonic cascade flows.
\end{abstract}

\subsection{Introduction}

To understand and predict flutter and forced response in turbomachinery blade rows, aeroelasticians require accurate and efficient models of the unsteady flow fields that arise from blade motion and incident gusts. Over the past two decades, many computational methods have been developed

\footnotetext{
${ }^{1}$ Assistant Professor, Department of Mechanical Engineering and Materials Science, Duke University, Durham, NC 27706.

${ }^{2}$ Research Assistant, Department of Mechanical Engineering and Materials Science, Duke University, Durham, NC 27706.
}

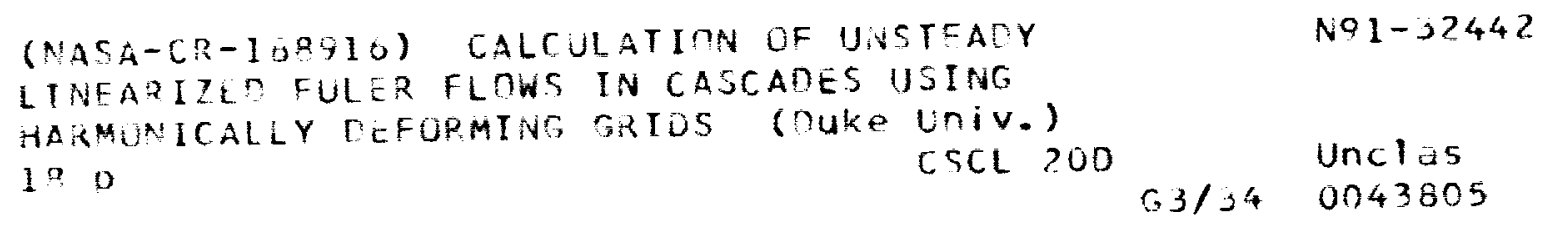

$N 91-32442$ 
to analyze unsteady flows in turbomachinery. For example, the linearized potential methods have been developed extensively [WG81, VC82, VC84, VU88, HV91]. Linearized potential analyses are computationally very efficient, and for aeroelastic applications, the small disturbance assumption is not a severe restriction. However, because of the inherent assumption of irrotational and isentropic flow, the linearized potential analyses are only valid for subsonic and low transonic speeds and flows without inlet swirl. To model the more complex flow fields found in transonic compressors and turbines, and as computer speeds and storage have improved, several time-accurate time-marching Euler [Hod84, FLM87, Gil88, HSR91] and Navier-Stokes [Rai89a, Rai89b, GH91] codes have been developed. These codes, unlike the linearized potential codes, account for both steady and unsteady vorticity and entropy. However, despite the recent advances in computer hardware, the time-marching approach is still computationally too expensive for routine design use.

Recently, several investigators have begun to develop linearized Euler analyses [NS76, HC89, HC91, LG91]. As in the linearized potential technique, the unsteady flow is assumed to be composed of a nonlinear mean flow plus a harmonically varying small perturbation flow. The unsteady perturbation flow is governed by the linearized Euler equations. Using this model of the flow accounts for entropy and vorticity generation and shocks, and permits analysis of unsteady flows about rotational nonisentropic mean flows. Furthermore, the solution of the linearized Euler equations, while not as efficient as solution of the linearized potential equations, is one to two orders of magnitude more efficient than computing the full nonlinear flow field while still retaining the essential physics of unsteady flows associated with forced response and the onset of flutter.

In this paper, a recently developed Euler analysis is presented. The linearized Euler equations are discretized and solved using a conservative pseudo-time time marching Lax-Wendroff scheme [NS76, Ni82, Dan87, HC91]. Two important features of the present analysis are the use of a deforming computational grid for flutter calculations, and shock capturing for transonic flow calculations. For flutter calculations, the use of a deforming computational grid eliminates large error producing gradient terms that would otherwise appear in the upwash boundary conditions and in the evaluation of the unsteady pressure on the airfoil surfaces. For transonic flow calculations, shock capturing is used to model the pressure impulse resulting from small harmonic motion of the shock. Shock capturing is considerably easier to implement than shock fitting. However, to predict correctly the unsteady loads due to the shock impulse, one must use a discretization scheme for the unsteady linearized flow solver that is a faithful linearization of the nonlinear flow solver [LG91]. Representative computational results are presented that demonstrate the capability of the method to predict unsteady subsonic and transonic flows. 


\subsection{Theory}

\subsubsection{The Linearized Euler Equations}

For compressible flows in which viscous forces may be neglected, the unsteady flow is governed by the Euler equations representing the conservation of mass, momentum, and energy, i.e,

$$
\frac{\partial \hat{\mathbf{U}}}{\partial t}+\frac{\partial \hat{\mathbf{F}}}{\partial \boldsymbol{x}}+\frac{\partial \hat{\mathbf{G}}}{\partial y}=0
$$

Here, $\hat{U}$ is the vector of conservation variables and $\hat{\mathbf{F}}$ and $\hat{\mathbf{G}}$ are the socalled flux vectors. These are given by

$$
\hat{\mathbf{U}}=\left[\begin{array}{c}
\hat{\rho} \\
\hat{\rho} \hat{u} \\
\hat{\rho} \hat{v} \\
\hat{e}
\end{array}\right], \hat{\mathbf{F}}=\left[\begin{array}{c}
\hat{\rho} \hat{u} \\
\hat{\rho} \hat{u}^{2}+\hat{p} \\
\hat{\rho} \hat{u} \hat{v} \\
\hat{\rho} \hat{u} \hat{h}_{0}
\end{array}\right], \hat{\mathbf{G}}=\left[\begin{array}{c}
\hat{\rho} \hat{v} \\
\hat{\rho} \hat{u} \hat{v} \\
\hat{\rho} \hat{v}^{2}+\hat{p} \\
\hat{\rho} \hat{v} \hat{h}_{0}
\end{array}\right]
$$

Where $\hat{\rho}$ is the static density, $\hat{u}$ and $\hat{v}$ are the $\boldsymbol{x}$ - and $\boldsymbol{y}$-components of the velocity, $\hat{p}$ is the pressure, $\hat{e}$ is the internal energy, and $\hat{h}_{0}$ is the total enthalpy.

Most previous linearized analyses have used computational grids fixed in space. To apply the upwash boundary condition at the airfoil surface, the boundary conditions must be extrapolated from the instantaneous position of the airfoil to the mean airfoil position, that is, to the boundary of the fixed grid. The additional extrapolation terms appearing in the upwash boundary condition contain velocity gradient terms that can be large and difficult to evaluate accurately, especially near the leading and trailing edges of the airfoils. Whitehead and Grant [WG81] overcame this difficulty for rigid-body airfoil motion by using a transformed velocity potential that can be viewed as equivalent to using a computational grid that undergoes rigid-body motion. Hall [Hal92] has recently developed a linearized potential analysis based on a variational principle that includes the effects of a grid undergoing arbitrary deformations thus allowing both rigid-body and flexible blade motion.

In the present linearized Euler analysis, we also make use of a deforming grid. The physical coordinates $x, y, t$ are related to the computational coordinates $\xi, \eta, \tau$ by the transformation

$$
\begin{aligned}
& x(\xi, \eta, \tau)=\xi+f(\xi, \eta, \tau) \\
& y(\xi, \eta, \tau)=\eta+g(\xi, \eta, \tau) \\
& t(\xi, \eta, \tau)=\tau
\end{aligned}
$$

The grid motion functions $f$ and $g$ are chosen so that the motion of the grid conforms to the motion of the airfoils. A typical example of unsteady 

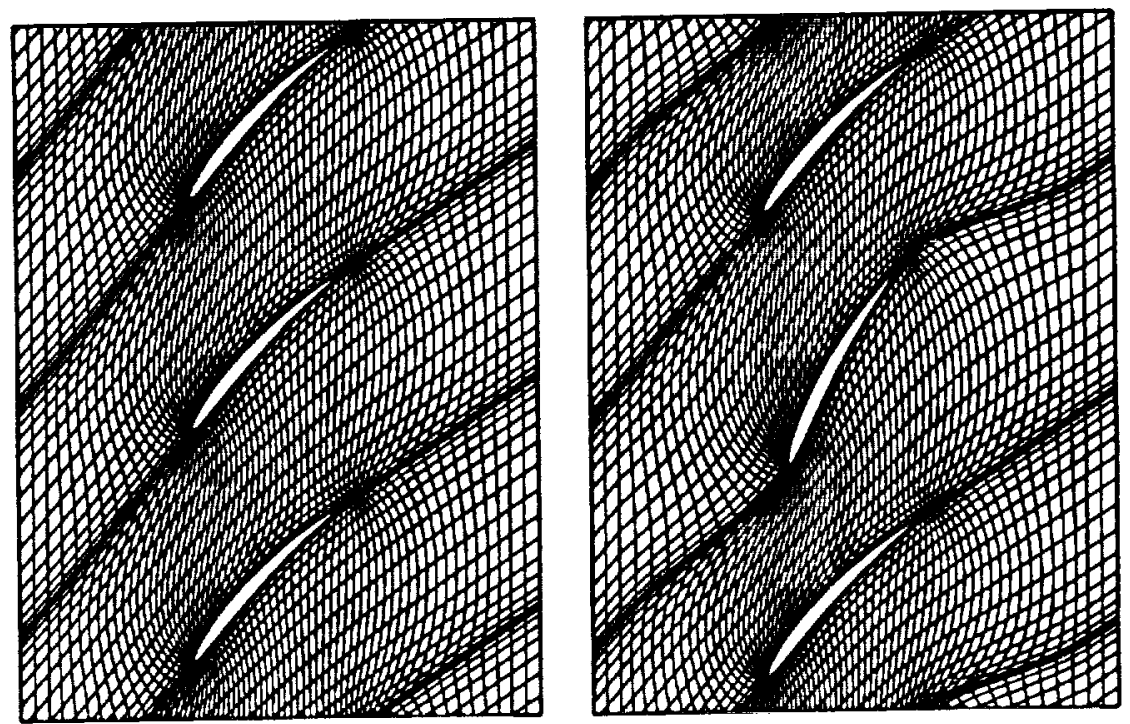

FIGURE 1.1. Left: Grid in computational coordinate system, $\xi, \eta$. Right: Grid in physical coordinate system, $x, y$, for the case of a cascade of airfoils plunging with an interblade phase angle, $\sigma$, of $90 \mathrm{deg}$. The cascade shown is Standard Configuration No. 10.

grid motion is shown in Fig. 1.1. Shown is a cascade of airfoils designated Standard Configuration No. 10 [Fra91] vibrating in pitch with an interblade phase angle, $\sigma$, of $90 \mathrm{deg}$. In the computational coordinate system, the airfoils and the grid appear to be stationary; in the physical domain, the airfoils and grid deform unsteadily.

Integrating the differential form of the Euler equations [Eq. (1.1)] over a control volume $D$ that is fixed in the computational coordinate system - but deforming in the physical coordinate system - and applying the divergence theorem gives the Euler equations in integral form, i.e.,

$$
\iint_{D} \frac{\partial}{\partial t} \hat{\mathbf{U}} d x d y+\oint_{\partial D}(\hat{\mathbf{F}} d y-\hat{\mathbf{G}} d x)=0
$$

With some manipulation, Eq. (1.5) can be expressed as

$$
\begin{gathered}
\frac{\partial}{\partial \tau} \iint_{D} \hat{\mathbf{U}} d x d y+\oint_{\partial D}\left[\left(\hat{\mathbf{F}}-\hat{\mathbf{U}} \frac{\partial f}{\partial \tau}\right) \frac{\partial y}{\partial \eta}-\left(\hat{\mathbf{G}}-\hat{\mathbf{U}} \frac{\partial g}{\partial \tau}\right) \frac{\partial x}{\partial \eta}\right] d \eta \\
+\oint_{\partial D}\left[\left(\hat{\mathbf{F}}-\hat{\mathbf{U}} \frac{\partial f}{\partial \tau}\right) \frac{\partial y}{\partial \xi}-\left(\hat{\mathbf{G}}-\hat{\mathbf{U}} \frac{\partial g}{\partial \tau}\right) \frac{\partial x}{\partial \xi}\right] d \xi=0
\end{gathered}
$$

Equation (1.6) is valid for both small and large disturbance unsteady flows. Large amplitude flows, which exhibit nonlinear behavior, could be 
computed by time marching Eq. (1.6). However, such computations are very expensive. Fortunately, for many flows of interest in aeroelastic applications, the unsteadiness in the flow is temporally harmonic and small compared with the mean flow. Therefore, to simplify the problem and also reduce the required computational time, we assume that the flow is decomposed into a zeroth-order mean or steady flow plus a forst-order unsteady perturbation flow. Hence,

$$
\hat{\mathbf{U}}(\xi, \eta, \tau)=\mathbf{U}(\xi, \eta)+\mathbf{u}(\xi, \eta) e^{j \omega \tau}
$$

Here, $\mathbf{U}$ is the vector of conservation variables representing the mean flow field, and $\mathbf{u}$ is the vector of small perturbation harmonic amplitudes of the conservation variables. The motion of the airfoils, and hence the grid, is assumed to be small and harmonic so that Eqs. (1.2)-(1.4) are replaced by

$$
\begin{aligned}
& x(\xi, \eta, \tau)=\xi+f(\xi, \eta) e^{j \omega \tau} \\
& y(\xi, \eta, \tau)=\eta+g(\xi, \eta) e^{j \omega \tau} \\
& t(\xi, \eta, \tau)=\tau
\end{aligned}
$$

where now $f$ and $g$ represent the small perturbation harmonic amplitudes of the grid motion.

Substitution of the perturbation assumptions into the nonlinear Euler equations, Eq. (1.6), and collection of terms of zeroth and first-order results in the mean flow Euler equations and the linearized Euler equations respectively. The mean flow Euler equations in integral form are given by

$$
\oint_{\partial D}(\mathbf{F} d \eta-\mathbf{G} d \xi)=0
$$

The linearized Euler equations are given by

$$
\begin{gathered}
j \omega \iint_{D} \mathbf{u} d \xi d \eta+\oint_{\partial D}\left(\frac{\partial \mathbf{F}}{\partial \mathbf{U}} \mathbf{u} d \eta-\frac{\partial \mathbf{G}}{\partial \mathbf{U}} \mathbf{u} d \xi\right)= \\
-j \omega \iint_{D} \mathbf{U}(d f d \eta+d \xi d g)+j \omega \oint_{\partial D}(f \mathbf{U} d \eta-g \mathbf{U} d \xi) \\
-\oint_{\partial D}(\mathbf{F} d g-\mathbf{G} d f)
\end{gathered}
$$

The Jacobian matrices $\partial \mathrm{F} / \partial \mathrm{U}$ and $\partial \mathrm{G} / \partial \mathrm{U}$ appearing in Eq. (1.12) are evaluated using the mean flow solution. For example, for an ideal gas with constant specific heats, one finds that the Jacobian $\partial F / \partial U$ is given by

$$
\frac{\partial \mathbf{F}}{\partial \mathbf{U}}=\left[\begin{array}{cccc}
0 & 1 & 0 & 0 \\
\frac{\gamma-3}{2} U^{2}+\frac{\gamma-1}{2} V^{2} & -(\gamma-3) U & -(\gamma-1) V & \gamma-1 \\
-U V & V & U & 0 \\
U\left(\frac{\gamma-1}{2} V_{T}^{2}-h_{0}\right) & h_{0}-(\gamma-1) U^{2} & -(\gamma-1) U V & \gamma U
\end{array}\right]
$$


where $V_{T}^{2}=U^{2}+V^{2}$.

The mean flow [described by (1.11)] is independent of the unsteady perturbation flow. The linearized Euler equations, Eq. (1.12), are linear, inhomogeneous, variable coefficient equations. The variable coefficients, i.e., the Jacobians, are functions of the mean flow field. The inhomogeneous portion of $\mathrm{Eq}$. (1.12), that is, the right-hand side, is a function of the mean flow and the prescribed grid motion.

\subsubsection{NEAR-Field Boundary Conditions}

\section{Periodicity}

Because the unsteady flow is governed by linear equations, the response of the cascade to blade motion or a gust may be found by decomposing the disturbance into a sum of travelling wave modes each with a different frequency, $\omega$, and interblade phase angle, $\sigma$. The total response of the cascade to the sum of modes is equal to the sum of the responses to each of the individual modes. Without loss of generality, we consider here a single travelling wave mode. The complex periodicity condition upstream and down stream of the cascade is then

$$
\mathbf{u}(\xi, \eta+G)=\mathbf{u}(\xi, \eta) e^{j \sigma}
$$

where $G$ is the blade-to-blade gap. This periodicity condition allows the computational domain to be reduced to a single blade passage.

Flow Tangency

The upwash boundary condition on the airfoil surface is

$$
\mathbf{v} \cdot \mathbf{n}=\left(j \omega \mathbf{r}+\mathbf{V} \cdot \tau \frac{\partial \mathbf{r}}{\partial s}\right) \cdot \mathbf{n}
$$

where $\mathbf{V}$ and $\mathbf{v}$ are the mean perturbation flow velocities, $\mathbf{n}$ and $\tau$ are unit vectors normal to and tangent to the mean airfoil surface, $s$ is the arc length along the airfoil surface, and $\mathbf{r}$ is the perturbation displacement of the airfoil surface. The two terms on the right-hand side represent the upwash due to the local translation and rotation of the airfoil surface. Note that because we use a deforming computational grid, no extrapolation terms are required.

\subsubsection{NUMERICAL INTEGRATION}

To integrate numerically the linearized Euler equations, we use the pseudotime time marching approach suggested by $\mathrm{Ni}$ and Sisto [NS76]. The unsteady perturbation amplitude $u$ is assumed to vary with time so that 
Eq. (1.7) is replaced by

$$
\hat{\mathbf{U}}(\xi, \eta, \tau)=\mathbf{U}(\xi, \eta)+\mathbf{u}(\xi, \eta, \tau) e^{j \omega \tau}
$$

Substitution of Eq. (1.16) into Eq.(1.6) and collection of terms of equal order results in the pseudo time-dependent linearized Euler equations,

$$
\frac{\partial}{\partial \tau} \iint_{D} \mathbf{u} d \xi d \eta+j \omega \iint_{D} \mathbf{u} d \xi d \eta+\oint_{\partial D}\left(\frac{\partial \mathbf{F}}{\partial \mathbf{U}} \mathbf{u} d \eta-\frac{\partial \mathbf{G}}{\partial \mathbf{U}} \mathbf{u} d \xi\right)=\mathbf{b}
$$

where $b$ is the same as the right-hand side of the original (time-invariant) linearized Euler equation, Eq. (1.12). Note that the first term on the lefthand side is the only term that does not appear in the original linearized Euler equation. This pseudo-time dependent term is driven to zero by time marching the equation to steady state.

Note the similarity of the linearized Euler equations, Eq. (1.17), to the nonlinear time-dependent Euler equations, Eq. (1.5). One advantage of the pseudo-time time-marching approach is that several nonlinear Euler solvers have been developed that can be applied with little modification to the linearized Euler equations. For example, the conservation variables $\tilde{U}$ in the nonlinear Euler solver are replaced by the perturbation conservation variables $\mathbf{u}$ in the linearized Euler solver. The nonlinear flux vectors $\hat{\mathbf{F}}$ and $\hat{\mathbf{G}}$ are replaced by the perturbation flux vectors $(\partial \mathbf{F} / \partial \mathbf{U}) \mathbf{u}$ and $(\partial \mathbf{G} / \partial \mathbf{U}) \mathbf{u}$. Furthermore, since only the steady state solution of the perturbation solution $\mathbf{u}$ is desired, acceleration techniques such as multi-grid and local time stepping may be used to reduce dramatically the required computational time.

For subsonic flow calculations, any consistent discretization of the linearized Euler equations is sufficient to produce consistent unsteady flow solutions. For transonic flows with shock capturing, however, the discretized perturbation equations should be a faithful linearization of the discretized unsteady nonlinear equations used to compute the steady flow [LG91]. In the present study, we use Ni's conservative finite volume implementation of the Lax-Wendroff method [Ni82, Dan87] to compute both the steady and unsteady flow fields. Local time stepping and Ni's multiple-grid acceleration technique are used to speed convergence in both the steady nonlinear Euler and unsteady linearized Euler codes. For subsonic flows, a fourthdifference smoothing operator is used to eliminate sawtooth modes. For transonic flows, a combination of fourth-difference and second difference smoothing is used to capture shocks.

\subsubsection{Far-Field Boundary Conditions}

Because the computational domain extends a finite distance in the axial direction (see Fig. 1.1), far-field boundary conditions are required at the far-field boundaries to prevent spurious reflections of outgoing waves. Previous investigators have found analytical expressions for the behavior of 
the linearized equations in the far field, and have matched these far-field solutions to the near-field numerical solutions at the upstream and downstream far-field boundaries. These analytical solutions were found for the special case of uniform mean flow in the far field. For transonic Euler flows, however, the mean flow in the far field is in general nonuniform. For example, the presence of a shock or multiple shocks in the blade passage will produce nonuniform total pressure in the downstream region. No general analytical expression has been developed for this situation.

To overcome this difficulty, the authors have developed an alternative treatment the far-field boundary conditions. Instead of finding the analytical eigenmodes of the linearized Euler equations in the far-field, we find the eigenmodes of the discretized linearized Euler equations. The numerical eigenmodes are then matched to the numerical near-field solution at the far-field boundaries. This procedure, although computationally less efficient, produces numerically exact nonreflecting boundary conditions. Reference [HC91] describes the implementation of these boundary conditions in more detail.

\subsection{Results}

\subsubsection{Transonic Channel Flow}

The first test case to be presented is of transonic flow through a diverging channel. This case is presented to demonstrate the ability of the linearized Euler method to model accurately shock motion using shock capturing.

The channel considered here has a height, $A$, given by

$$
A(x)=A_{\text {inlet }}\left\{1.10313+.10313 \tanh \left[5\left(x-\frac{1}{2}\right)\right]\right\}, \quad 0 \leq x \leq 1
$$

So that the results can be compared to a one-dimensional shock-fitting theory, $A_{\text {inlet }}$ is taken to be small compared with the channel length $\left(A_{\text {inlet }}=0.01\right)$. The inflow total pressure, $P_{T}$, total density, $\rho_{T}$, and flow velocity, $U$, are $1.0,1.364$, and 1.0 , respectively. The back pressure, $P_{\text {exit }}$ is 0.7422 . Shown in Fig. 1.2 is the Mach number and pressure distribution as computed by the present nonlinear steady Euler solver using a $129 \times 5$ node computational grid. Also shown for comparison is the solution found using a steady quasi-one-dimensional, shock-fitting, Euler solver using 1001 grid nodes in the $x$ direction. Note the excellent agreement between the two approaches. The only noticeable differences occur at the shock where the present nonlinear Euler solver smears the shock over about five grid nodes.

Next, we consider the case of a quasi-steady perturbation in the back pressure. The perturbation solution was computed using three different methods. We first computed the solution using the present linearized solver; then using a quasi one-dimensional, shock-fitting, linearized Euler solver; 


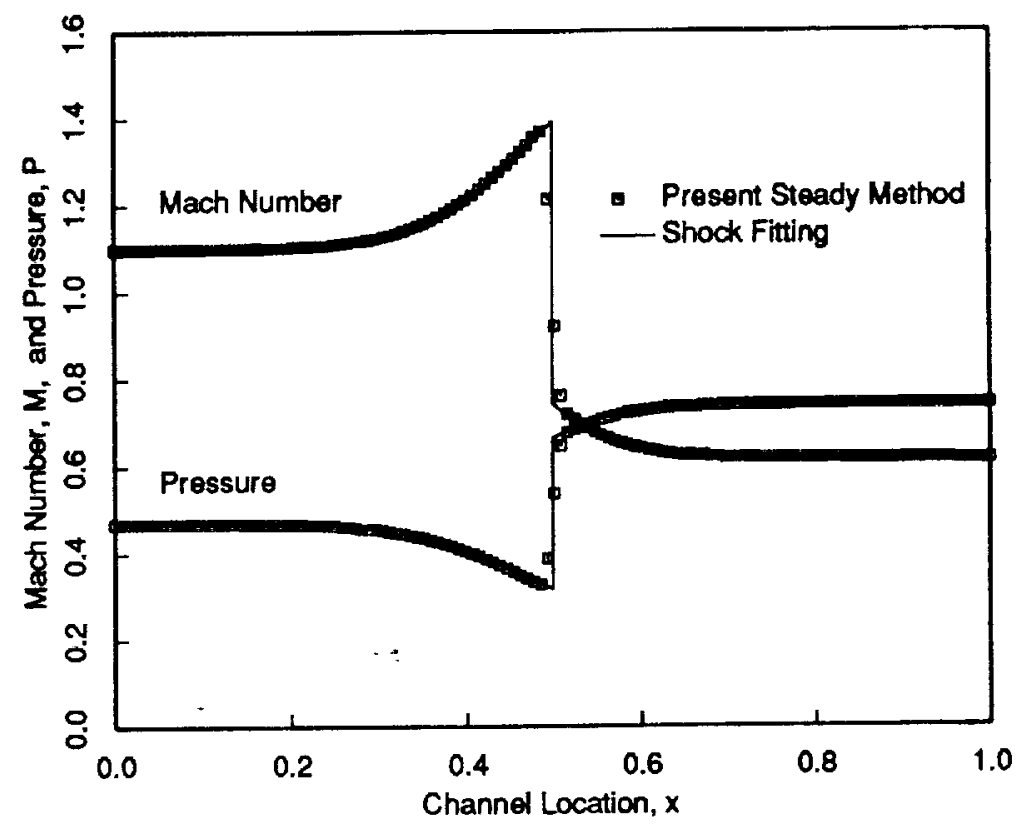

FIGURE 1.2. Steady transonic flow in a diverging channel.

and finally, by differencing the solutions found using the present nonlinear steady Euler solver at two slightly different back pressures. The results are shown in Fig. 1.3. The three solutions are seen to be in very good agreement away from the shock. At the shock, the two shock-capturing techniques produce an impulse of pressure. This impulse represents the load exerted on the channel walls due to the displacement of the shock. Note that the present linearized Euler solution is nearly identical to the differenced nonlinear solutions.

We now consider the case of an unsteady variation in the back pressure. Shown in Fig. 1.4 are the computed real and imaginary parts of the unsteady pressure distribution due to a unit perturbation in the back pressure with an excitation frequency, $\omega$, of 1.0 . The results of the present linearized Euler code agree well with the quasi one-dimensional, shock-fitting, linearized Euler solver.

To determine whether the present linearized Euler solver correctly predicts the unsteady loads induced by the motion of the shock, we integrated the pressure along the lower wall to obtain the "wall force." These results are tabulated in Table 1.1 for several different excitation frequencies. Also given in Table 1.1 is the wall force computed using the shock-fitting code. For our purposes, the grid is sufficiently fine that the shock-fit solution 

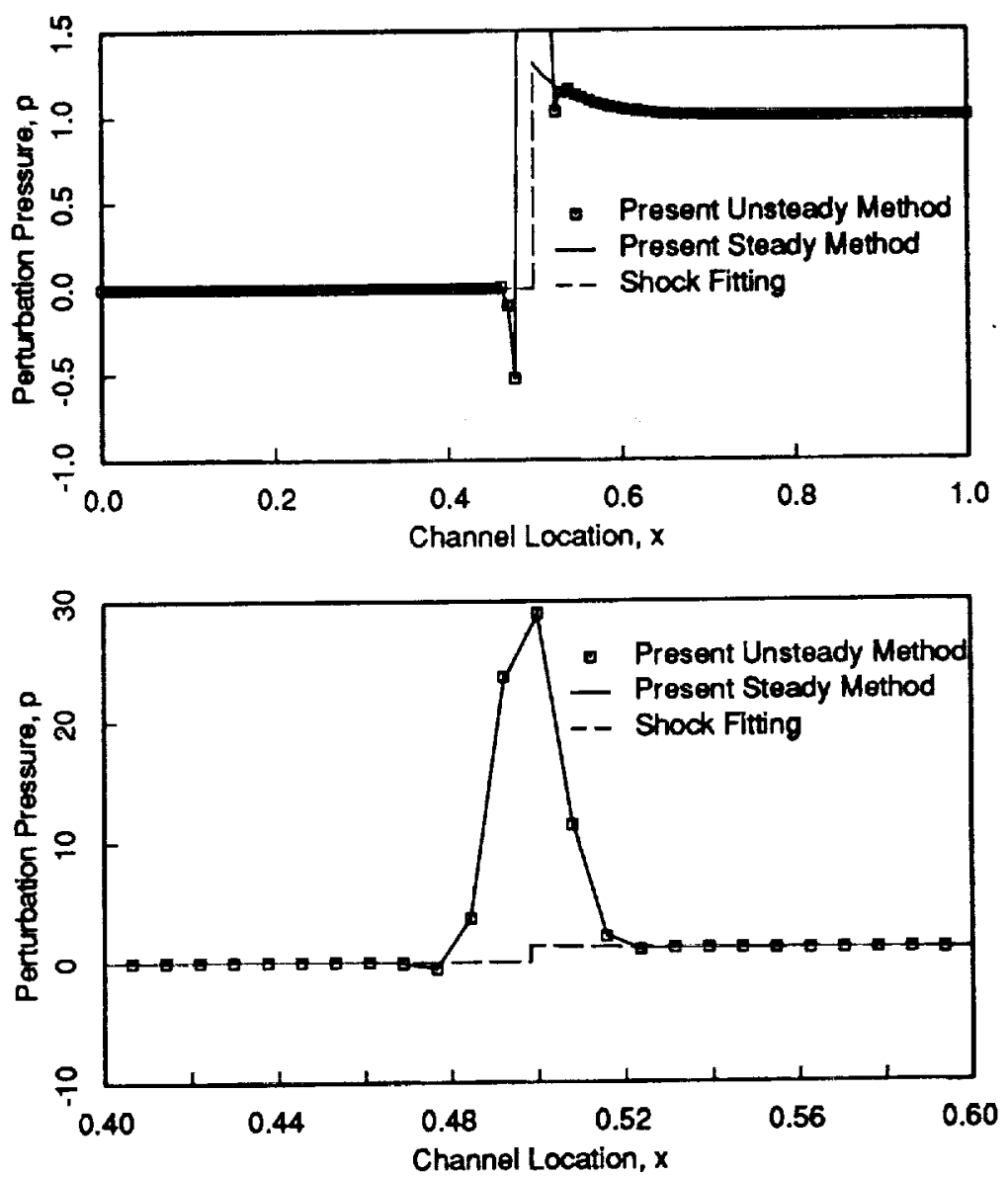

FIGURE 1.3. Perturbation pressure in a diverging channel due to a steady perturbation in back pressure. Bottom figure shows detail of shock impulse.

may be considered exact. For low frequencies, the two codes are in almost perfect agreement suggesting that the shock impulse found using shock capturing is properly modelled. For the higher frequency cases, the agreement is still good, but there is a slight error in the phase of the wall force. We believe that these differences are due to dispersion errors in regions away from the shock rather than a limitation in shock capturing at high frequencies. Finally, For the $\omega=1$ case, we deliberately introduced two types of inconsistency into the linearized Euler solver to determine their influences on the solutions. In the first case, we used a different level of smoothing in the linearized unsteady Euler code than was used in the nonlinear steady 


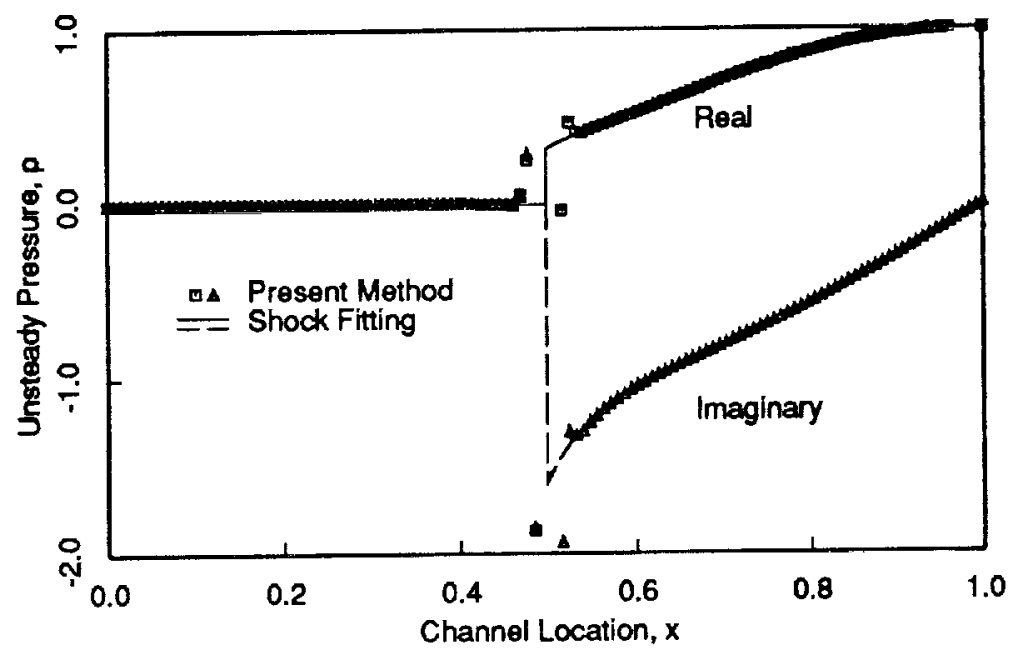

FIGURE 1.4. Unsteady pressure in a diverging channel due to an unsteady perturbation in back pressure. The reduced frequency, $\bar{\omega}$, based on channel length and inflow velocity is 1.0 .

Euler code. In the second case, global time stepping was used in the steady Euler code and local time stepping was used in the linearized unsteady code. In both cases, the inconsistency between steady and unsteady codes is seen to increase the error in the unsteady wall force. The errors are much more pronounced, however, for the latter case. These results are consistent with the claim by Lindquist and Giles that the linearized code should be a faithful linearization of the nonlinear code [LG91].

\subsubsection{Subsonic Flow in a COMPRESSOR}

Having demonstrated the accuracy of the method for transonic channel flow, we next consider unsteady flows in a compressor geometry to demonstrate the ability of the method to accurately compute the unsteady flow about a cascade of loaded airfoils. The cascade considered is the newly designated Standard Configuration No. 10 [Fra91]. The airfoils have a circular arc camber distribution with a maximum height of 5 percent of the chord with a slightly modified NACA 0006 thickness distribution. The stagger angle, $\Theta$, is $45 \mathrm{deg}$ and the gap-to-chord ratio, $G$, is 1.0. For the subsonic case considered here, the steady inflow angle, $\Omega_{-\infty}$, is $55 \mathrm{deg}$ and the inflow Mach number, $M_{-\infty}$, is 0.7 . Under these conditions, the flow is entirely subsonic with a maximum Mach number on the suction surface of about 0.92 .

Figure 1.5 shows the computed unsteady pressure distribution for the case where the airfoils pitch about points near their midchords with an 
TABLE 1.1. Predicted pressure loads in a transonic diverging channel due to an unsteady perturbation in back pressure.

\begin{tabular}{clcc}
\hline \hline Frequency, $\omega$ & \multicolumn{1}{c}{ Scheme } & Wall Force & Shock Force \\
\hline 0.0 & 1D Shock Fitting $^{\text {Nonlinear Euler }}{ }^{a}$ & $1.0305+0.0000 j$ & $0.5133+0.0000 j$ \\
& Present Method $^{2.0326+0.0000 j}$ & - \\
& $1.0320+0.0000 j$ & - \\
\hline 0.5 & 1D Shock Fitting & $0.6866+0.6115 j$ & $0.1796-0.4426 j$ \\
& Present Method & $0.6863+0.6100 j$ & - \\
\hline 1.0 & 1D Shock Fitting & $0.1249-0.6267 j$ & $-0.2543-0.2797 j$ \\
& Present Method $^{b}$ & $0.1293-0.6222 j$ & - \\
& Present Method $^{b}$ & $0.1315-0.6184 j$ & - \\
& Present Method $^{c}$ & $0.1778-0.6133 j$ & - \\
\hline 2.0 & 1D Shock Fitting & $-0.0807-0.1801 j$ & $-0.1182+0.1667 j$ \\
& Present Method & $-0.0750-0.1831 j$ & - \\
\hline \hline
\end{tabular}

${ }^{a}$ Results from the steady analysis were found for two slightly different back pressures. The two solutions were then differenced and normalized by $\Delta P_{\text {exit }}$.

${ }^{b}$ Smoothing coefficient in ateady analysis is 0.15 ; smoothing coefficient in unsteady analysis is 0.30 . ysis.

Global time stepping used in ateady analysia; local time stepping used in unsteady anal-

interblade phase angle, $\sigma$, of $90 \mathrm{deg}$ and a reduced frequency, $\bar{\omega}$, of 0.5 (Standard Configuration No. 10, Case 2). Here, the pressure is nondimensionalized by the upstream steady flow quantity $\rho V_{T}^{2}$. To assess the accuracy of the analysis, solutions were computed on both a $65 \times 17$ and a $129 \times 33$ node grid. The good agreement between the coarse and fine grid solutions indicates that the fine grid solution is nearly grid converged. Also shown for comparison is the solution found using a deforming grid linearized potential method [Hal92]. Note that the solutions are well behaved in the neighborhood of the leading and trailing edges. The use of a deforming grid greatly improves the accuracy of the solution in these regions.

The linearized Euler solver is very efficient. The unsteady solution computed on the $65 \times 17$ node grid required 565 seconds of computer time on an IBM RISC System $/ 6000$ model 320 computer. For comparison, the steady solution required 110 seconds.

Having computed the unsteady pressure distribution on the airfoil surface, one can integrate to obtain the pitching moment. Shown in Figure 1.6 is the imaginary part of the pitching moment computed for a range of interblade phase angles, $-180 \leq \sigma \leq 180$, at a reduced frequency, $\bar{\omega}$, of 1.0. Also shown is the pitching moment computed using Hall's deforming grid linearized potential method. The peaks in the solution are acoustic resonances. We were unable to obtain converged solutions near one of the upstream acoustic resonance points $(\sigma=117.1 \mathrm{deg})$. Note that there is generally good agreement between the linearized Euler and linearized potential 

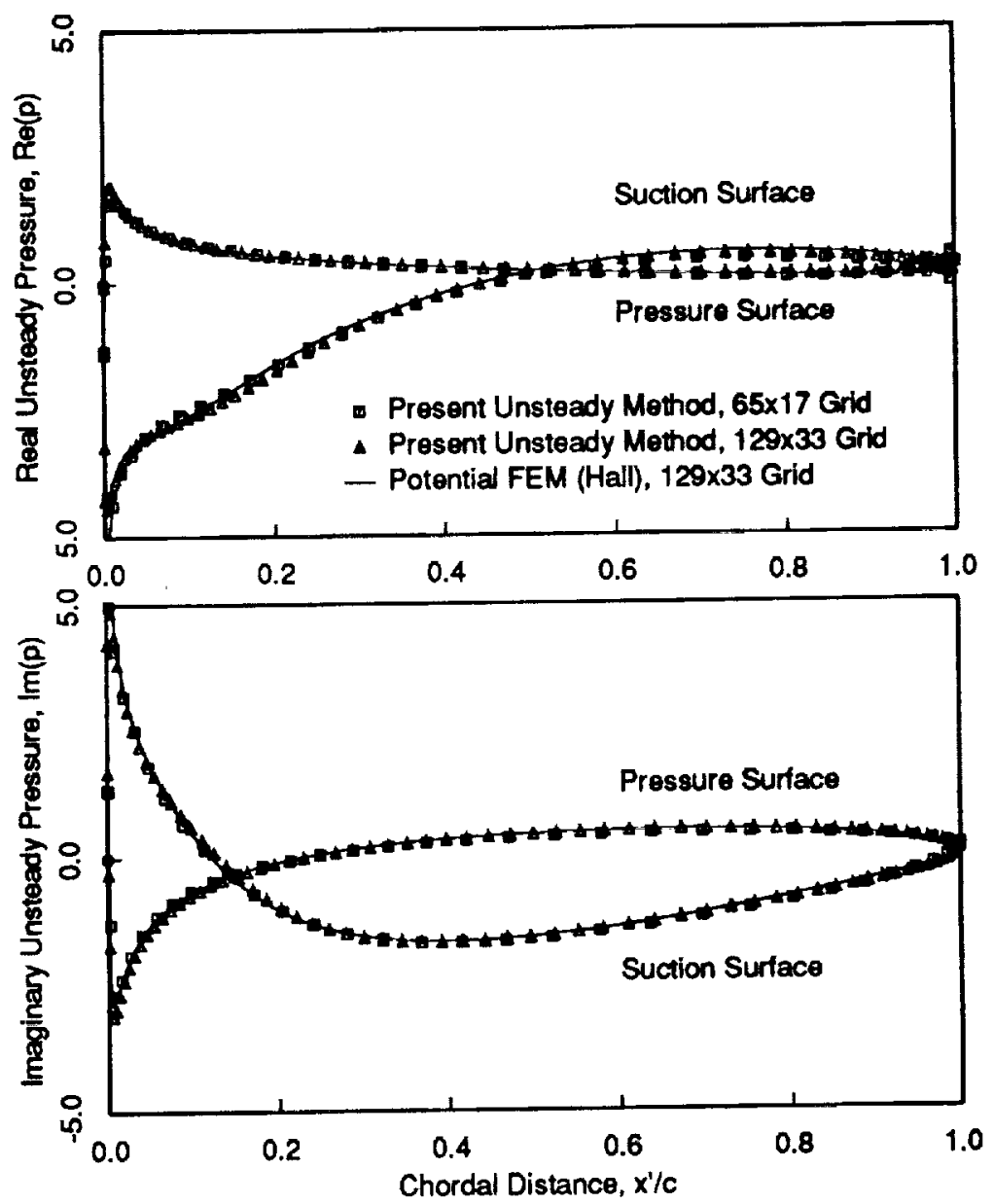

FIGURE 1.5. Unsteady pressure on surface of subsonic compressor blade due to pitching motion (Standard Configuration No. 10, Case 2). The reduced frequency, $\omega$, is 0.5 , and the interblade phase angle, $\sigma$, is $90 \mathrm{deg}$.

solution. One noticeable difference is seen at $\sigma=-20 \mathrm{deg}$. This point is very near one of the upstream acoustic resonance points $(\sigma=-26.93)$. Nevertheless, the good agreement elsewhere in the superresonant regions demonstrates the effectiveness of the numerical far-field boundary conditions. 


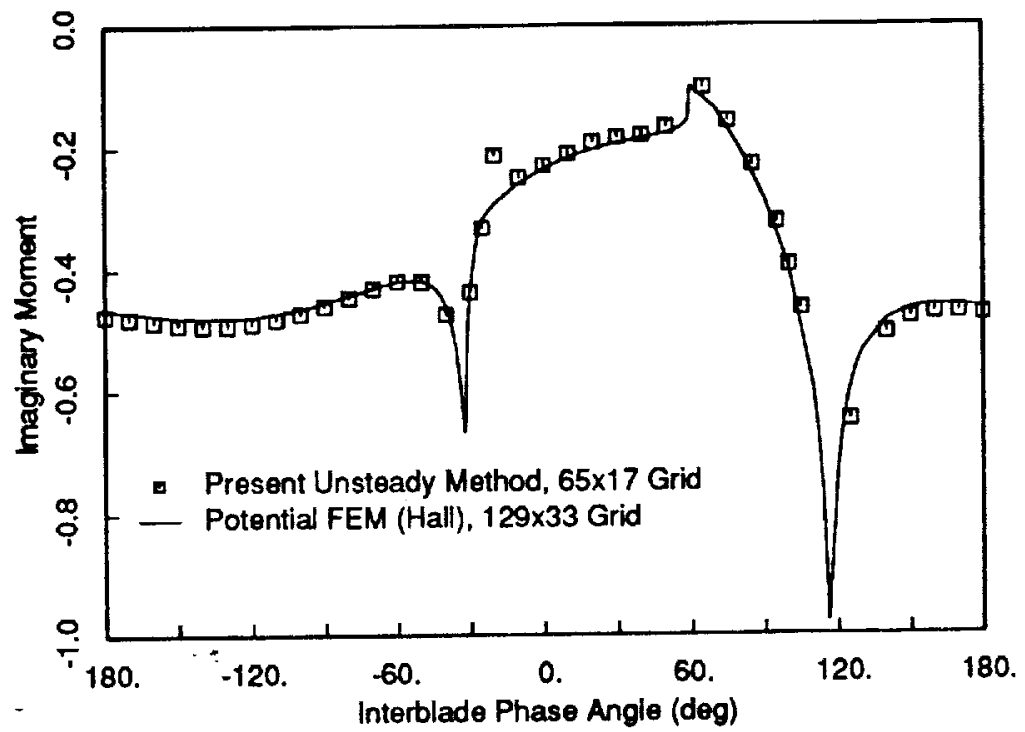

FIGURE 1.6. Imaginary pitching moment acting on subsonic compressor blades due to pitching motion (Standard Configuration No. 10). The reduced frequency, $\bar{\omega}$, is 1.0 .

\subsubsection{Transonic Flow in a COMPREsSor}

The final case to be considered here is flow through a transonic compressor. The cascade is again Standard Configuration No. 10. However, the flow conditions are such that there is a supersonic patch on the suction surface of the airfoil. The mean inflow angle, $\Omega_{-\infty}$, is $58 \mathrm{deg}$ and the inflow Mach number, $M_{-\infty}$, is 0.8. Shown in Fig. 1.7 is the computed isentropic Mach number distribution on the surface of the airfoil. Also shown is the Mach number distribution computed using a full potential technique [VC84, VU88]. Note that for the solution computed with the present nonlinear Euler solver, the shock is smeared over about five grid points.

Having computed the steady flow solution, we consider the case of the airfoils vibrating in pitch with an interblade phase angle, $\sigma$, of $90 \mathrm{deg}$ with a reduced frequency, $\bar{\omega}$, of 1.0 (Standard Configuration No. 10, Case 20 ). Figure 1.8 shows the computed unsteady pressure distribution on the airfoil surface. The shock impulse representing the unsteady load due to unsteady shock motion is clearly visible on the suction surface. Also shown is the pressure distribution computed using Verdon's linearized potential code, LINSUB code [VU88]. The linearized Euler and linearized potential solutions agree quite well on the pressure surface. However, the agreement is not as good on the suction surface, particularly ahead of the shock. This 


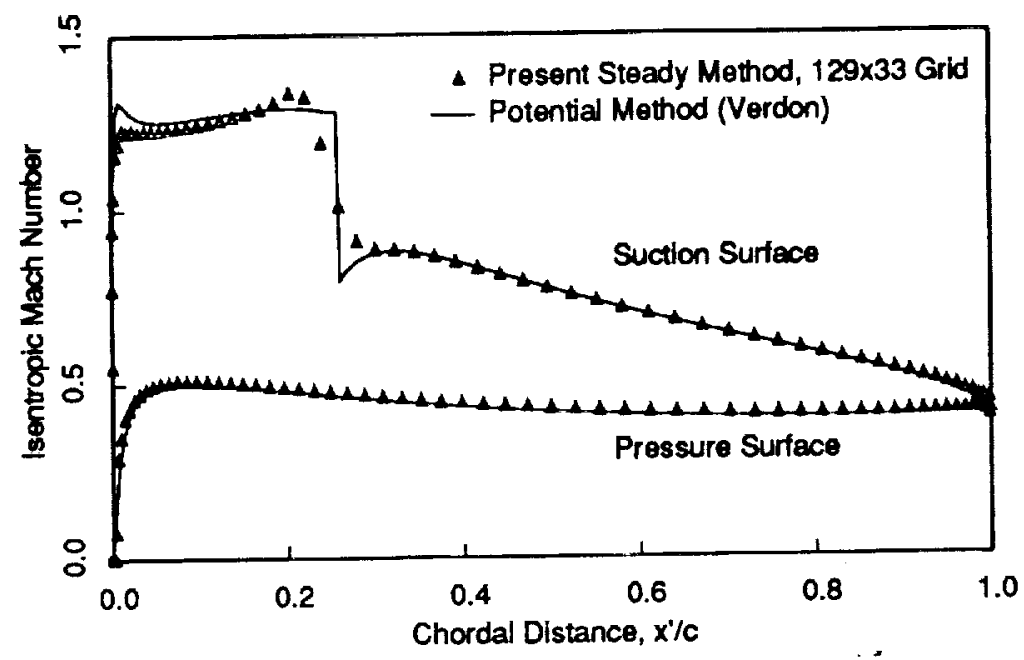

FIGURE 1.7. Steady pressure distribution on transónic compressor blade (Standard Configuration No. 10). The inflow Mach number, $M$, is 0.8 , the gap-to-chord ratio, $G$, is 1.0 , the stagger angle, $\theta$, is $45 \mathrm{deg}$., and the inflow angle, $\Omega_{-\infty}$, is 58 deg.

difference could arise from neglecting entropy and vorticity generation in the steady and unsteady potential analyses.

\subsection{Summary}

A two-dimensional linearized Euler solver has been developed that is capable of computing unsteady small disturbance flows in cascades. Two important features of the analysis are the use of a deforming grid to improve the accuracy of flutter calculations, and the use of shock capturing to model shock motion. Using the pseudo-time time-marching technique, one can solve the linearized equations with any of a number of well known schemes originally developed to solve the nonlinear Euler equations. Furthermore, since only the steady state harmonic perturbation solution is desired, convergence acceleration techniques may be used to speed convergence. Results presented in the paper clearly demonstrate the ability of the analysis to predict unsteady subsonic and transonic flows accurately and efficiently.

Acknowledgments: This work was supported by a grant from NASA Lewis Research Center, NASA Grant NAG3-1192, with Dr. Daniel Hoyniak serving as technical monitor. The authors wish to acknowledge helpful discus- 

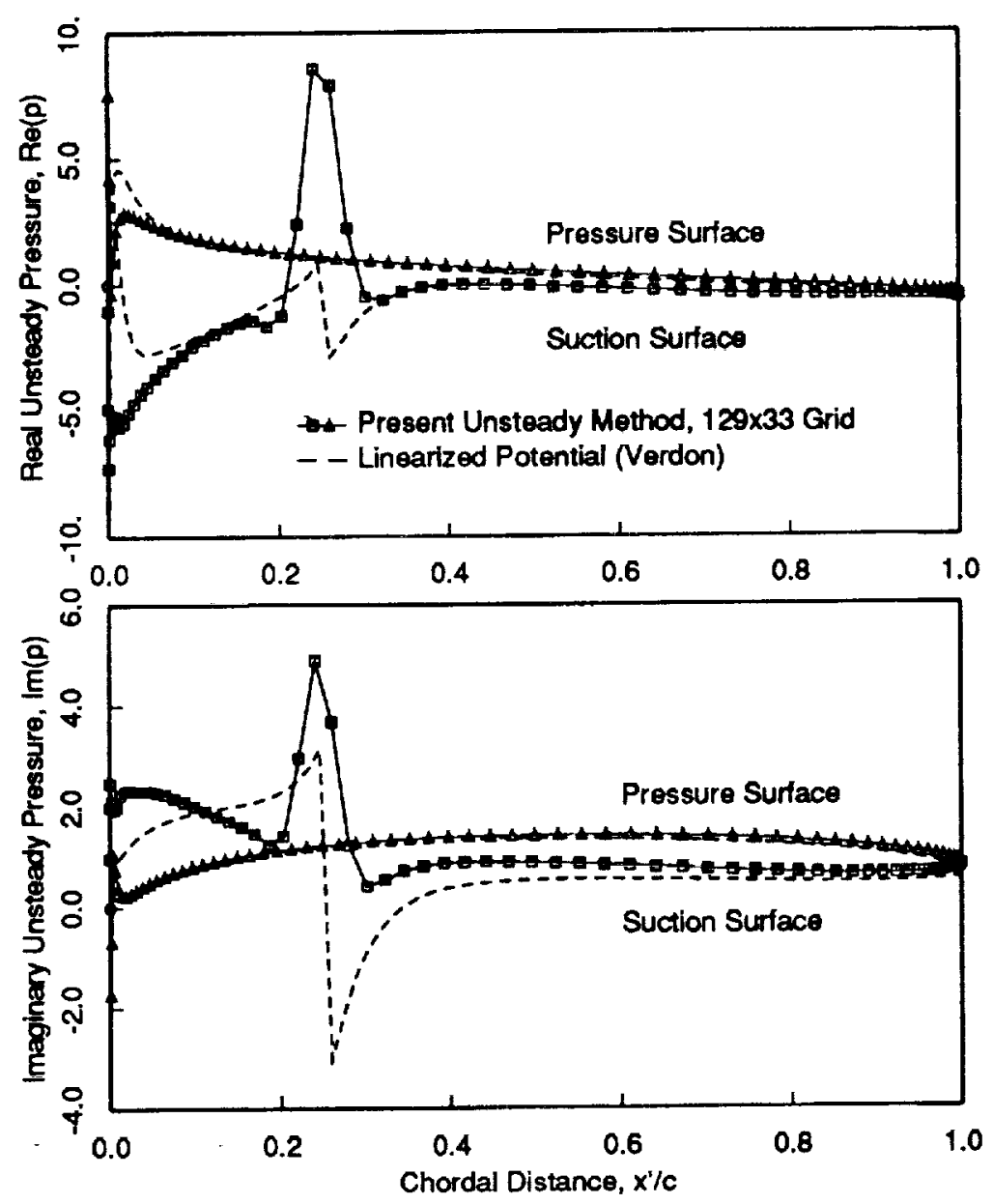

FIGURE 1.8. Unsteady pressure on surface of transonic compressor blade due to pitching motion (Standard Configuration No. 10, Case 20). The reduced frequency, $\bar{\omega}$, is 1.0 , and the interblade phase angle, $\sigma$, is $90 \mathrm{deg}$.

sions with Dr. Graham Holmes, Dr. Michael Giles, and Ms. Dana Lindquist. In addition the authors are indebted to Dr. Joseph M. Verdon for providing unsteady pressure distribution predictions for Standard Configuration No. 10. 


\subsection{REFERENCES}

[Dan87] J. D. Dannenhoffer, III. Grid Adaptation for Complex TwoDimensional Transonic Flows. ScD thesis, Massachusetts Institute of Technology, Cambridge, Massachusetts, August 1987.

[FLM87] A. Fourmaux and A. Le Meur. Computation of unsteady phenomena in transonic turbines and compressors. In H.E. Gallus and S. Servaty, editors, Unsteady Aerodynamics and Aeroelasticity of Turbomachines and Propellers, Proceedings of the Fourth International Symposium, pages 87-108, Aachen, West Germany, September 6-10, 1987.

[Fra91] T. H. Fransson, June 1991. Private communication.

[GH91] M. B. Giles and R. Haimes. Validation of a numerical method for unsteady flow calculations. ASME paper 91-GT-271, presented at the International Gas Turbine and Aeroengine Congress and Exposition, Orlando, Florida, June 3-6, 1991.

[Gil88] M. B Giles. Calculation of unsteady wake/rotor interaction. AIAA Journal of Propulsion, 4(4):356-362, July-August 1988.

[Hal92] K. C. Hall. A deforming grid variational principle and finite element method for computing unsteady small disturbance flows in cascades. AIAA Paper 92-0655, to be presented at the AIAA 30th Aerospace Sciences Meeting, Reno, Nevada, January 6-9, 1992.

[HC89] K. C. Hall and E. F. Crawley. Calculation of unsteady flows in turbomachinery using the linearized Euler equations. AIA A Journal, $27(6): 777-787$, June 1989.

[HC91] K. C. Hall and W. S. Clark. Prediction of unsteady aerodynamic loads in cascades using the linearized Euler equations on deforming grids. AIAA Paper 91-3378, presented at the AIAA/SAE/ASME/ASEE 27th Joint Propulsion Conference, Sacramento, California, June 24-26, 1991.

[Hod84] H. P. Hodson. An inviscid blade-to-blade prediction of a wake generated unsteady flow. ASME paper 84-GT-43, 1984.

[HSR91] D. L. Huff, T. W. Swafford, and T. S. R. Reddy. Euler flow predictions for an oscillating cascade using a high resolution wave-split scheme. ASME paper 91-GT-198, presented at the International Gas Turbine and Aeroengine Congress and Exposition, Orlando, Florida, June 3-6, 1991.

[HV91] K. C. Hall and J. M. Verdon. Gust response of a cascade operating in a nonuniform mean flow. AIAA Journal, 29(9):1463-1471, September 1991. 
[LG91] D. R. Lindquist and M. B. Giles. On the validity of linearized unsteady Euler equations with shock capturing. AIAA Paper 811598-CP, presented at the AIAA 10th Computational Fluid Dynamics Conference, Honolulu, Hawaii, June 24-27, 1991.

[Ni82] R. H. Ni. A multiple-grid scheme for solving the Euler equations. AIA A Journal, 20(11):1565-1571, November 1982.

[NS76] R. H. Ni and F. Sisto. Numerical computation of nonstationary aerodynamics of flat plate cascades in compressible flow. Transactions of the ASME: Journal of Engineering for Power, 98:165-170, April 1976.

[Rai89a] M. M. Rai. Three-dimensional Navier-Stokes simulations of turbine rotor-stator interaction; part I-methodology. AIA A Journal of Propulsion and Power, 5(3):307-311, May-June 1989.

[Rai89b] M. M. Rai. Three-dimensional Navier-Stokes simulations of turbine rotor-stator interaction; part II - results. AIAA Journal of Propulsion and Power, 5(3):312-319, May-June 1989.

[VC82] J. M. Verdon and J. R. Caspar. Development of a linear unsteady aerodynamic analysis for finite-deflection subsonic cascades. AIAA Journal, 20(9):1259-1267, September 1982.

[VC84] J. M. Verdon and J. R. Caspar. A linearized unsteady aerodynamic analysis for transonic cascades. Journal of Fluid Mechanics, 149:403-429, December 1984.

[VU88] J. M. Verdon and W. J. Usab, Jr. Advances in the numerical analysis of linearized unsteady cascade flows. Technical Report UTRC Report R88-957685-1, United Technologies Research Center, 1988.

[WG81] D. S. Whitehead and R. J. Grant. Force and moment coefficients of high deflection cascades. In P. Suter, editor, Proceedings of the 2nd International Symposium on Aeroelasticity in Turbomachines, pages $85-127$, Zurich, 1981. Juris-Verlag. 